\title{
Development and beta test of a smokeless tobacco cessation program for firefighters
}

\author{
Nattinee Jitnarin' ${ }^{1}$, Walker S. C. Poston', Sara A. Jahnke', Christopher K. Haddock', Herbert H. Severson ${ }^{2}$
}

\begin{abstract}
INTRODUCTION The prevalence of smokeless tobacco (SLT) use among firefighters is substantially higher than the general population and similar occupational groups. Despite the significant health risks associated with SLT and its impact on occupational readiness, there are no occupationally-tailored SLT education or treatment programs for the fire service. The purpose of this study was to beta test QUIT SPIT!, a self-help SLT cessation program that is culturally tailored for the US fire service and firefighters who are interested in quitting.

METHODS After development and tailoring the QUIT SPIT! SLT cessation program for firefighters, the feasibility and acceptability of the program were evaluated in a sample of eleven SLT-using firefighters who wanted to quit. The primary outcome was a 7-day point prevalence of SLT abstinence measured at 4 and 12 weeks post-enrollment follow-up assessments.

RESULTS Four firefighters reported having quit SLT (7-days point prevalence) at follow-up at 12 weeks. Those who did not achieve SLT abstinence reported reductions in frequency and quantity in SLT use and demonstrated a decrease in nicotine dependence. Firefighters also reported being satisfied with the QUIT SPIT! cessation program.

CONCLUSIONS The results provide strong support for the feasibility and acceptability of the QUIT SPIT! in SLT-using firefighters interested in quitting. The findings provide critical information about the next steps for further development and evaluation of the QUIT SPIT! program.
\end{abstract}

AFFILIATION

1 Center for Fire, Rescue and EMS Health Research, NDRI-USA, Leawood, United States 2 Oregon Research Institute, Eugene, United States

CORRESPONDENCE TO Nattinee Jitnarin. Center for Fire, Rescue and EMS Health Research, NDRI-USA, 1920 West 143rd Street, Leawood, KS 66224,United States. Email: jitnarin@ndri-usa.org

\section{KEYWORDS}

occupational health, firefighters, smokeless tobacco, fire service, behavioral intervention, smokeless tobacco cessation

Received: 2 September 2020 Revised: 25 January 2021 Accepted: 31 January 2021

\section{INTRODUCTION}

Over the past decade, the fire service demonstrated an exceptionally high prevalence of smokeless tobacco (SLT) use $(11-18 \%)^{1-4}$. The rates were more than double those found in the general male population $(7 \%)^{5}$, and were comparable to other occupational groups with the highest rates of use (i.e. construction workers, miners $)^{6,7}$. Several reasons for firefighters' increasing use of SLT were documented ${ }^{8,9}$, including indoor smoking and disease presumption rules in fire departments, national fire service organizations' efforts to promote smoking cessation, and targeted marketing by the smokeless tobacco industry.
However, there are no occupationally-tailored SLT treatment programs for the fire service and no published reports in the literature of efforts to address this significant issue. This lack of resources exists despite the high rate of SLT use among firefighters, the strong association between SLT use and several cancers with elevated rates among firefighters ${ }^{10,11}$, and the significant relationships between multiple types of cancer and firefighting ${ }^{12}$.

Our study developed and evaluated a self-help SLT cessation program culturally-tailored for the US fire service - entitled QUIT SPIT! - to help SLTusing firefighters quit successfully. The purpose 
of the project was to develop, tailor, and evaluate a self-help SLT cessation program that was based on a previously developed and empirically validated program called 'Enough Snuff'13-15. We chose to modify and tailor Enough Snuff for firefighters because the program had been evaluated with military personnel, and there are many cultural similarities between the fire service and the military. For instance, the fire service operates under a paramilitary structure, shares cultural traditions with the military, and has a relatively high rate of former military members among its ranks ${ }^{16,17}$. We also believed QUIT SPIT! would be cost-effective, and easily disseminated to any fire department, and it contained cessation program elements that have proven to be effective for SLT cessation.

Our goal was to develop and tailor QUIT SPIT! to meet the cultural and unique needs of SLTusing firefighters who want to quit. In this study, we present findings from the beta test of the QUIT SPIT! intervention and evaluate the effect of using this program with a convenience sample of eleven firefighters who reported daily use of SLT and wanted to quit.

\section{METHODS}

\section{Program/intervention development}

Our culturally-tailored self-help SLT cessation program - entitled QUIT SPIT! - is a 54-page booklet of combined graphics and text to assist firefighters in quitting using SLT with a step-by-step approach to quitting, detailed instructions demonstrating effective cessation methods, and information to help firefighters dealing with withdrawal and staying abstinent. The intervention was developed to be costeffective and designed to be easily disseminated to fire departments.

Prior to implementation, we collected data and obtained feedback to develop and tailor the intervention to this population. We used a combination of key-informant interviews (i.e. fire service leaders and firefighters who were former and current SLT users) and the existing scientific literature to develop and tailor the empiricallyvalidated Enough Snuff intervention ${ }^{13,18}$ for firefighters. Social Cognitive Theory (SCT) ${ }^{19}$ was used as a framework for intervention development ${ }^{20,21}$. SCT posits that learning occurs through: 1) modeling of recognizable peers (e.g. other firefighters); 2) observing positive consequences for desired behaviors and negative outcomes for continuing negative behaviors (e.g. SLT use); and 3) self-efficacy (e.g. the belief that they have the ability to follow through with desired behavior).

We tailored, revised, and refined QUIT SPIT! specifically for use with firefighters and to ensure its suitability for their culture based on data collected from the interviews and feedback to identify and address fire service's specific challenges to cessation (i.e. occupational stress, SLT use acceptability). The materials were organized around four key steps to quitting: 1) Get Ready, evaluating readiness and motivation to quit; 2) Plan to Quit, setting a quit date and selecting a quit plan; 3) Quitting, providing techniques and solutions on dealing with withdrawal symptoms; and 4) Stay Quit, providing resources on maintaining quit status.

\section{Step 1: Get Ready}

Firefighters learned how to quantify and evaluate their SLT use, assess their nicotine dependence, and determine their degree of readiness to quit. Also included in this step were sample testimonials from those who quit successfully to help firefighters identify and share their motives or intentions for quitting. Firefighters were encouraged to write down their motives or reasons for quitting, which were then used as reminders to maintain motivation.

\section{Step 2: Plan to Quit}

This step contained several quit approaches and methods such as 'cold turkey' or nicotine reduction approaches that firefighters could choose to fit their SLT-use behavior and lifestyle. Once they made a plan for quitting, they were encouraged to share their intention to quit on a specific date with others (e.g. spouse/partner, crew or chief) who could support their quitting effort. Testimonials and a brief list of 'dos and don'ts' for the support person were included to help guide the supporter to be positive and avoid 'nagging' and negative behaviors.

\section{Step 3: Quitting}

This step focused on ways to help firefighters through the cessation process by providing them 
with information related to withdrawal symptoms. It prepared firefighters to deal with situations they can anticipate once they start the quitting process. Lists of symptoms experienced during SLT withdrawal, resources and other support materials, including text and graphic information on pharmacotherapy and alternative options for dealing with nicotine withdrawal, were also provided.

\section{Step 4: Staying Quit}

The final step aimed to help firefighters prepare and plan for the difficult situations and their urge to chew after quitting. It included on- and off-duty scenarios that firefighters may find associated with their urges. Examples of on-duty situations included being around or working with other SLT users while anticipated off-duty situations included examples such as relaxing after a meal or watching television. This section also provided messages of encouragement, and testimonials from other firefighters who had quit successfully, and supportive messages addressed to those who had relapsed and encouraged them to go back to and redo the previous steps.

Once developed, QUIT SPIT! was reviewed by leading experts in tobacco cessation areas for consistency with the overall tobacco cessation guidelines and by fire service personnel to improve its cultural appropriateness. These reviews resulted in minor editing of the content and language of the QUIT SPIT!

\section{Pilot testing}

Pilot testing was conducted in eleven career firefighters who used SLT and wanted to quit in a large Midwestern city using convenience sampling procedure. The pilot was conducted between August 2019 and January 2020 by investigators who traveled in-person to the fire stations for all assessments. At baseline, firefighters consented to and completed the surveys. They were provided QUIT SPIT! program enrollment information and received a demonstration of the components. Firefighters were encouraged to set goals and make an action plan for quitting. They were revisited at 4 and 12 weeks after their baseline to obtain follow-up measures. Investigators encouraged firefighters to interact with the program components as much as desired at times convenient to their lifestyle. At the follow-up at 12 weeks, participants self-reported their experience using the QUIT SPIT! program. Questions included their opinion of the program's overall helpfulness and usefulness for their SLT reduction or abstinence, and ratings of satisfaction and ease of program use. Firefighters were asked about how the treatment could be improved, barriers or challenges encountered while using the program, and their perceptions of best methods for integrating technology into the dissemination of QUIT SPIT!

Measurement items included: a self-reported 7-day point prevalence abstinence based on the question - 'In the past 7 days, have you ever used chewing tobacco, snuff, or dip?'; amount SLT use in quantity (i.e. number of cans/pouches used per week), and frequency (i.e. number of dips/chews per day); and level of tobacco dependence. This intervention was low-intensity because it was selfdirected, and investigators did not interact with the participants after the initial introduction at the baseline. The research protocol was approved by the NDRI-USA, Inc. Institution Review Board.

\section{RESULTS}

The majority of participants were Caucasian and were at the rank of 'firefighter' (Table 1). On average, they were $39.0(\mathrm{SD}=8.8)$ years old and had served fire service for 15.1 years. Participants had used SLT for approximately 18.6 years $(\mathrm{SD}=8.5)$ before participating in this study and reported that they used an average of 11.7 dips per day $(\mathrm{SD}=8.1)$. Of the 11 firefighters enrolled, one discontinued study participation prior to the follow-up at 12 weeks. Four firefighters reported having quit SLT ( 7-days point

\section{Table 1. Baseline characteristics of participants ( $N=11)$}

\begin{tabular}{ll} 
Characteristics & \\
\hline Age (years), Mean (SD) & $39.0(8.8)$ \\
Time in fire service (years), Mean (SD) & $15.1(9.5)$ \\
Caucasian, \% (n) & $90.9(10)$ \\
Cans/pouches of SLT use per week, Mean (SD) & $4.6(3.7)$ \\
Dips/chews of SLT per day, Mean (SD) & $11.7(8.1)$ \\
Regular SLT use (years), Mean (SD) & $18.6(8.5)$ \\
Exclusive SLT users, \% (n) & $81.8(9)$ \\
Dual use with e-cigarette, \% (n) & $9.1(1)$ \\
Poly use with cigarette and e-cigarette, \% (n) & $9.1(1)$
\end{tabular}


prevalence) at assessment at 12 weeks. For firefighters who did not achieve SLT abstinence results, they reported reductions in cans/pouches per week or having fewer chews per day between baseline and assessment at 12 weeks.

Feedback from the firefighters regarding the QUIT SPIT! program was overwhelmingly positive. They agreed that the program was useful and helped to motivate them to quit SLT. They also stated that the program increased their knowledge of SLT's health risk and cessation methods. Those who did not quit successfully at the end of the intervention stated that their SLT use behavior changed because of the program, such as decreasing SLT use frequency, increasing their use-interval duration, or using herbal snuff as a replacement of SLT. Firefighters also reported that these behavior changes increased their confidence in maintaining reduction and continuing the quitting process. Specific suggestions for improvement were compiled and used for planning the next version of the program to be adapted for a web-based or smartphone application-based program to improve accessibility and delivery. The suggestions included: offering more testimonial or stories of firefighters who successfully quit SLT; adding graphic elements to make the educational sections more interesting; providing calendar or progress timeline to track the related tasks or assignments associated with each quitting step, and having fire service organizations such as the union involved to help to disseminate the program. Firefighters also reported that they would recommend the program to another firefighter who currently used SLT.

\section{DISCUSSION}

Despite the high prevalence of SLT use among fire service personnel and their unique job characteristics, no SLT cessation programs have been previously developed or tailored for this occupational group. This study is the first to determine the feasibility and acceptability of an SLT cessation program that was culturally-tailored for the US fire service. The current findings also provided insight into firefighters' preferences for receiving the SLT cessation program. The results suggest that QUIT SPIT! is an appropriate and acceptable SLT cessation intervention for firefighters who want to quit.

\section{Strengths and limitations}

Our study's strengths include the use of participants from the targeted population of firefighters who used SLT and wanted to quit, and an SLT cessation program tailored to their unique occupational culture. Behavioral interventions for tobacco cessation designed for a specific cultural group produce significantly better results and are more effective than interventions that are not culturally tailored ${ }^{22,23}$. The information obtained from pilot participants can increase the depth and validity of the pilot evaluation. Thus, this information can, in turn, be used to refine the delivery of QUIT SPIT! and its treatment components and for developing a prototype QUIT SPIT! app among firefighters and for implementing the intervention on a larger study in the next research steps.

Limitations include the small sample size, the lack of a control group, and the inability to test for statistical significance in the changes after intervention. However, the small sample size was justified for this study as the goal was to evaluate the feasibility and acceptability of QUIT SPIT! prior to a larger pilot trial. Also, biochemical verification of self-reported SLT use was not conducted. However, previous research in population-based surveys ${ }^{24-26}$ and among firefighters ${ }^{1-3}$ has found self-report to be a valid and reliable measure of tobacco use.

\section{CONCLUSIONS}

A self-help SLT cessation program culturally-tailored for the US fire service - QUIT SPIT! - is feasible and acceptable among firefighters who used SLT and wanted to quit. It can be easily distributed to any fire department as a stand-alone program or used as a complement to any existing tobacco cessation program used in the fire service. The findings from this study will be used to inform the refinement of QUIT SPIT! and provide support for a larger randomized trial. Thus, the next step of this research will be developing and adapting the QUIT SPIT! into an app-based program, then conducting a pilot randomized controlled trial to examine its ability to influence SLT cessation outcomes in this population.

\section{REFERENCES}

1. Haddock CK, Jitnarin N, Poston WS, Tuley B, Jahnke $\mathrm{SA}$. Tobacco use among firefighters in the central 
United States. Am J Ind Med. 2011;54(9):697-706. doi:10.1002/ajim.20972

2. Jitnarin N, Poston WS, Haddock CK, Jahnke SA, Day RS. Tobacco use pattern among a national firefighter cohort. Nicotine Tob Res. 2015;17(1):66-73. doi:10.1093/ntr/ntu131

3. Jitnarin N, Haddock CK, Poston WS, Jahnke S. Smokeless tobacco and dual use among firefighters in the central United States. J Environ Public Health. 2013;2013:1-7. doi:10.1155/2013/675426

4. Jitnarin N, Poston WSC, Haddock CK, Jahnke SA, Day RS, Severson HH. Prevalence and Correlates of Late Initiation of Smokeless Tobacco in US Firefighters. Nicotine Tob Res. 2017;20(1):130-134. doi:10.1093/ntr/ntw321

5. Hu SS, Homa DM, Wang T, et al. State-Specific Patterns of Cigarette Smoking, Smokeless Tobacco Use, and E-Cigarette Use Among Adults — United States, 2016. Prev Chronic Dis. 2019;16:180362. doi:10.5888/pcd16.180362

6. Graber JM, Delnevo CD, Manderski MT, et al. Cigarettes, Smokeless Tobacco, and Poly-Tobacco Among Workers in Three Dusty Industries. J Occup Environ Med. 2016;58(5):477-484. doi:10.1097/JOM.0000000000000699

7. Mazurek JM, Syamlal G, King BA, Castellan RM, Division of Respiratory Disease Studies, National Institute for Occupational Safety and Health, CDC. Smokeless tobacco use among working adults - United States, 2005 and 2010. MMWR Morb Mortal Wkly Rep. 2014;63(22):477482. PMID:24898164.

8. Jahnke SA, Poston WS, Jitnarin N, Haddock CK. Health concerns of the U.S. fire service: perspectives from the firehouse. Am J Health Promot. 2012;27(2):111-118. doi:10.4278/ajhp.110311-QUAL-109

9. Poston WS, Haddock CK, Jitnarin N, Jahnke SA. A national qualitative study of tobacco use among career firefighters and department health personnel. Nicotine Tob Res. 2012;14(6):734-741. doi:10.1093/ntr/ntr282

10. Cogliano V, Straif K, Baan R, et al. Smokeless tobacco and tobacco-related nitrosamines. Lancet Oncol. 2004;5(12):708. doi:10.1016/s1470-2045(04)01633-x

11. Richter P, Hodge K, Stanfill S, Zhang L, Watson C. Surveillance of moist snuff: total nicotine, moisture, $\mathrm{pH}$, un-ionized nicotine, and tobacco-specific nitrosamines. Nicotine Tob Res. 2008;10(11):1645-1652. doi:10.1080/14622200802412937

12. Guidotti TL. Evaluating causality for occupational cancers: the example of firefighters. Occup Med (Lond). 2007;57(7):466-471. doi:10.1093/occmed/kqm031

13. Severson HH, Gordon JS. Enough Snuff: Quitting Smokeless Tobacco: A Guide for Military Personnel. 2nd ed. ETR Associates; 2010.

14. Severson HH. Population Health Trial for Smokeless Tobacco Cessation with Military Personnel. Eugene, OR: Oregon Research Institute; 2004. https://apps.dtic.mil/ dtic/tr/fulltext/u2/a425921.pdf. Accessed July 18, 2020.

15. Severson HH, Peterson AL, Andrews JA, et al. Smokeless tobacco cessation in military personnel: a randomized controlled trial. Nicotine Tob Res. 2009;11(6):730-738. doi:10.1093/ntr/ntp057

16. Military Service and Firefighting. Military.com. https:// www.military.com/veteran-jobs/career-advice/militarytransition/transition-to-firefighting-from-militaryservice.html. Accessed July 27, 2020.

17. Byrne D. The Military is a Good First Step to Becoming a Firefighter. Firehouse. https://www.firehouse.com/ careers-education/article/12158326/firefighter-jobshow-a-military-background-can-help-with-fire-servicecareers. Published January 13, 2016. Accessed July 27, 2020.

18. Severson HH, Gordon JS. Enough snuff: a guide for quitting smokeless tobacco. 8th ed. Scotts Valley, CA: ETR Associates; 2010.

19. Bandura A. Social Foundations of Thought and Action: A Social Cognitive Theory. Englewood Cliffs, NJ: Prentice Hall; 1986.

20. Côté-Arsenault D, Morrison-Beedy D. Maintaining your focus in focus groups: avoiding common mistakes. Res Nurs Health. 2005;28(2):172-179. doi:10.1002/nur.20063

21. Patton MQ. Qualitative Research \& Evaluation Methods : Integrating Theory and Practice. 4th ed. Thousand Oaks, CA: Sage; 2015.

22. Haddad LG, Al-Bashaireh AM, Ferrell AV, Ghadban R. Effectiveness of a Culturally-Tailored Smoking Cessation Intervention for Arab-American Men. Int J Environ Res Public Health. 2017;14(4):411. doi:10.3390/ijerph14040411

23. Lipkus IM, Lyna PR, Rimer BK. Using tailored interventions to enhance smoking cessation among AfricanAmericans at a community health center. Nicotine Tob Res. 1999;1(1):77-85. doi:10.1080/14622299050011181

24. Connor Gorber S, Schofield-Hurwitz S, Hardt J, Levasseur G, Tremblay M. The accuracy of self-reported smoking: a systematic review of the relationship between selfreported and cotinine-assessed smoking status. Nicotine Tob Res. 2009;11(1):12-24. doi:10.1093/ntr/ntn010

25. Velicer WF, Prochaska JO, Rossi JS, Snow MG. Assessing outcome in smoking cessation studies. Psychol Bull. 1992;111(1):23-41. doi:10.1037/0033-2909.111.1.23

26. Wong SL, Shields M, Leatherdale S, Malaison E, Hammond D. Assessment of validity of self-reported smoking status. Health Rep. 2012;23(1):1-7. PMID:22590805.

\section{ACKNOWLEDGMENTS}

The authors would like to thank the firefighters for their support and participation in the research.

\section{CONFLICTS OF INTEREST}

The authors have each completed and submitted an ICMJE form for disclosure of potential conflicts of interest. The authors declare that they have no competing interests, financial or otherwise, related to the current work. All authors report grants from the American Cancer Society during the conduct of the study. 


\section{FUNDING}

This work was supported by the American Cancer Society (Grant number: 129326-MRSG-1610401-CPPB).

\section{AUTHORS' CONTRIBUTIONS}

All authors contributed to the conception and design of the study. $\mathrm{NJ}$ performed the qualitative and statistical analysis and drafted the manuscript. NJ and WP conducted data collection. All authors read and approved the final manuscript.

PROVENANCE AND PEER REVIEW

Not commissioned; externally peer reviewed. 